\title{
Detección temprana de fracasos a tratamiento en pacientes con tuberculosis pulmonar.
}

\author{
Early detection in treatment failure in patients with pulmonary tuberculosis \\ Bernabé Ortiz Antonio ${ }^{1}$. \\ RESUMEN
}

Objetivo: Identificar factores asociados a fracaso de terapia en pacientes con tuberculosis pulmonar durante los 2 primeros meses de tratamiento específico, basados en el peso, el índice de masa corporal (IMC) y el esputo. Materiales y Métodos: Estudio retrospectivo de tipo caso-control apareado por sexo y edad ( \pm 2 años), llevado a cabo en Pampas de San Juan de Miraflores. Todas los fracasos a tratamiento entre el año 2000 y junio del 2005 con frotis positivo fueron incluidos como casos; y los pacientes que ingresaron al programa con prueba de esputo positiva al inicio, y que cumplieron el criterio de cura del programa fueron los controles, 2 por cada caso. Resultados: Veintiocho casos fueron apareados con 56 controles. Los factores asociados a fracaso fueron: al inicio del tratamiento, el antecedente de tratamiento previo (OR = 3,54, IC 95\%: 1,22-10,26). Al mes de tratamiento, la pérdida de IMC $(\mathrm{OR}=10,65$, IC 95\%: 2,02-56,01) estuvo asociado a fracaso a tratamiento, mientras que al segundo mes de seguimiento, la presencia de esputo positivo (OR = 25,58, IC 95\%: 2,10-311,27) y la pérdida de IMC (OR = 7,08, IC 95\%: 1,06-47,55) estuvieron asociados. Ningún control tuvo frotis positivo en o después del tercer mes de tratamiento. Conclusiones: Este estudio muestra que una parte de los pacientes que desarrollaran fracaso a tratamiento podrían detectarse durante el mismo, especialmente mediante el antecedente de tratamiento previo, la variación del IMC y el resultado positivo de esputo al segundo mes. Estos hallazgos podrían mejorar el diagnóstico de formas resistentes y la iniciación de un tratamiento apropiado. (Rev Med Hered 2007;18:123-128).

PALABRAS CLAVE: Fracaso a tratamiento antituberculoso, frotis, índice de masa corporal, peso.

\section{SUMMARY}

Objective: To identify factors associated with therapy failure in patients with pulmonary tuberculosis during the first two months of specific treatment, based on weight, body mass index (BMI) and sputum. Materials and Methods: A retrospective case-control study matched by sex and age ( \pm 2 years) was undertaken in Pampas de San Juan de Miraflores. All therapy failures between 2000 and June 2004 with smear-positive results were included as cases; while patients who had a positive sputum result at the treatment beginning and were declared as cured by the program criteria were controls, 2 per each case. Results: Twenty eight cases were matched with 56 controls. Factors associated with treatment failure included: at the therapy beginning, previous treatment history $(\mathrm{OR}=3.54$,

${ }^{1}$ Unidad de Epidemiología. Universidad Peruana Cayetano Heredia. Magíster en Control de Enfermedades Infecciosas y Tropicales. Universidad Peruana Cayetano Heredia. Lima, Perú. 
95\% IC: 1.22-10.26). At the first treatment month, body mass index loss (OR = 10.65, 95\% IC: 2.02-56.01); whilst at the second month of treatment, a smear-positive result $(\mathrm{OR}=25.58,95 \% \mathrm{IC}: 2.10-311.27)$ and $\mathrm{BMI}$ loss $(\mathrm{OR}=$ 7.08, 95\%IC: 1.06-47.55) were associated. Any control had a positive sputum result after third month of treatment. Conclusions: This study shows that some patients who will have therapy failure could be detected during treatment; especially by means of the previous treatment history, variation of BMI and smear-positive result at the second month. These findings could improve the multi-drug resistant tuberculosis (MDR-TB) diagnosis and prompt initiation of appropriate treatment.(Rev Med Hered 2007;18:123-128).

KEY WORDS: Tuberculosis treatment failure, smear, body mass index, weight.

\section{INTRODUCCIÓN}

La Estrategia Nacional para Control de la Tuberculosis en el Perú, define fracaso a esquema primario o secundario como un concepto bacteriológico, que debe ser confirmado a través de un cultivo positivo (1). En pacientes que reciben tratamiento en forma estrictamente supervisada, debe sospecharse fracaso en aquellos que mantienen baciloscopías positivas hasta el cuarto mes de tratamiento o en los que presentan baciloscopías positivas después de un periodo de negativización de dos meses $(2,3)$.

Sin embargo, estudios previos demuestran que se debe sospechar fracaso según la evolución clínica y de laboratorio (frotis de esputo) del paciente (4-6); sin necesidad de esperar hasta el cuarto mes de tratamiento para corroborarlo. Además, algunos reportes han documentado que los pacientes que fracasan al tratamiento tienen alta probabilidad de tener formas multi-resistentes (MDR) de la enfermedad $(7,8)$. Siendo así, el hecho de esperar por amplio margen de tiempo, pone en riesgo la salud del paciente y de los contactos, debido a que las formas resistentes de la enfermedad tienen mal pronóstico y son difíciles de tratar, sin tomar en cuenta los costos que produce.

Aunque la estrategia DOTS ha sido una de las mejores herramientas usadas por el programa de control para el marcado descenso de la incidencia de tuberculosis en nuestro país (9), esta se ve seriamente amenazada por la aparición de formas resistentes de tuberculosis (10).

El objetivo del estudio fue identificar factores asociados al fracaso al tratamiento antituberculoso durante la terapia específica, centrado en la evolución del esputo, el peso y el índice de masa corporal (IMC) de los pacientes durante los dos primeros meses de seguimiento.

\section{MATERIALES Y MÉTODOS}

Estudio observacional retrospectivo de tipo caso- control, realizado en Pampas de San Juan de Miraflores, zona urbano marginal de Lima.

Los datos de los pacientes fueron tomados del seguimiento que realiza el Programa de Control de Tuberculosis en la Micro-red de Pampas de San Juan, desde enero del 2000 hasta Junio del 2005, independiente de si eran pacientes nuevos o con antecedente de tratamiento previo.

Los casos fueron definidos como aquellos pacientes que fueron admitidos en el programa para tratamiento antituberculoso con resultado de esputo positivo al inicio del mismo y que mantenían resultados de esputo positivo después de cuatro meses de tratamiento o se tornaban positivos después de un periodo de negativización de 2 meses, siendo confirmados por cultivo. Los controles, 2 por cada caso, eran pacientes que ingresaron al programa con prueba de esputo positiva al inicio, apareados por edad y sexo ( \pm 2 años) y que cumplieron el criterio de cura del programa, es decir tenían al menos un frotis de esputo negativo al finalizar el tratamiento.

Para comparación entre casos y controles se usó la evolución mensual de los pacientes a través de la prueba de esputo, el peso y el índice de masa corporal (IMC) en los 2 primeros meses de terapia.

Los datos fueron digitados en una base creada en Microsoft Excel versión 2000, siendo posteriormente trasladadas para el análisis estadístico a STATA 8.0 para Windows. La prueba de U de Mann-Whitney fue usada para comparación de variables numéricas, mientras que las categóricas fueron evaluadas a través de la prueba exacta de Fisher.

La variación del peso al primer mes de terapia se calculó como una diferencia entre el peso al primer mes y el peso inicial. Similarmente se hizo para el segundo mes de tratamiento y para el IMC. Posteriormente, estas variables fueron categorizadas usando el cero como punto de corte. De esta manera, se generó dos grupos, unos que tenían una diferencia menor a cero (pérdida de peso o IMC) y los otros que tenían una diferencia 
igual o mayor a cero (no pérdida de peso o IMC) según el caso.

Finalmente, se evaluaron los riesgos (OR) a través del análisis de regresión logística y se construyeron los modelos bivariado y multivariado para fracaso al tratamiento con un nivel de confianza del 95\%. Cada posible factor fue evaluado en su momento correspondiente, por lo que se construyeron tres modelos multivariados, al inicio, al primer $\mathrm{y}$, al segundo mes de tratamiento. Asimismo, todos los resultados presentados fueron ajustados por edad y sexo.

\section{RESULTADOS}

Durante el periodo de estudio, se encontraron 28 pacientes que cumplían con el criterio de fracaso al tratamiento establecido previamente, los cuales se emparejaron con 56 controles de un total de 366 potenciales controles existentes durante el periodo de estudio. No existió diferencia entre casos y controles con respecto al tiempo de duración del tratamiento ni a la irregularidad del mismo.

Dentro de las características al inicio del tratamiento, el único factor asociado a fracaso al tratamiento fue el antecedente de haber recibido tratamiento antituberculoso previo $(p=0,03)$. Las características iniciales se muestran en la tabla $\mathrm{N}^{\circ} 1$.

En la tabla $\mathrm{N}^{\circ} 2$ se muestra la comparación de las variables usadas para el seguimiento. Se encontró diferencia estadísticamente significativa entre el promedio del peso al primer mes $(\mathrm{p}=0,02)$, pero no al segundo mes $(p=0,18)$ de tratamiento. Sin embargo, se encontró marcada diferencia entre el promedio del IMC al primer $(\mathrm{p}=0,003)$ y al segundo mes de evolución $(p=0,01)$. Cuando el seguimiento fue evaluado según las pruebas de esputo, no se halló diferencia estadísticamente significativa entre casos y controles al primer mes $(p=0,06)$, pero sí al segundo mes de tratamiento ( $p=0,001)$. Ningún control tuvo frotis positivo en o después del tercer mes de tratamiento (Tabla $\mathrm{N}^{\circ} 2$ ).

El análisis multivariado al inicio del tratamiento mostró que el antecedente de tratamiento previo fue el único factor asociado a fracaso a tratamiento $(\mathrm{OR}=3,54$, IC 95\%: 1,22 - 10,26). El modelo multivariado al primer mes de tratamiento reveló que aquellos que perdieron IMC tuvieron mayor riesgo de fracaso $(\mathrm{OR}=10,65$, IC 95\%: 2,02 - 56,01), mientras que el modelo multivariado al segundo mes de tratamiento demostró que la presencia de examen de esputo positivo (OR = 25,58, IC 95\%: 2;10 - 311,27) y la pérdida de IMC durante los 2 meses de seguimiento (OR $=7,08$, IC 95\%: 1,06 - 47,55) estuvieron asociados a fracaso a tratamiento tuberculoso (Tabla $\mathrm{N}^{\circ} 3$ ).

\section{DISCUSIÓN}

La falla a tratamiento es una amenaza económica y de salud, tanto porque el paciente continúa siendo un foco infeccioso en la comunidad y puede conducir a la diseminación de formas resistentes, como por la pérdida económica atribuida a la ausencia e incapacidad para trabajar (11).

Aunque existen estudios que demuestran que la persistencia de exámenes de esputo positivo no debe ser considerado como un criterio diagnóstico de fracaso a tratamiento específico en los países desarrollados $(12,13)$; en países en desarrollo como el nuestro, donde a veces no existen los medios económicos necesarios

Tabla $N^{\circ} 1$. Características iniciales de la población de estudio.

\begin{tabular}{lcccc}
\hline \multicolumn{1}{c}{ Variable } & & Casos & Controles & p \\
\hline \multirow{2}{*}{ Sexo } & Masculino & $17(60,71 \%)$ & $34(60,71 \%)$ & 1 \\
Antecedente de & Femenino & $11(39,29 \%)$ & $22(39,29 \%)$ & \\
tratamiento previo & Negativo & $17(60,71 \%)$ & $47(83,93 \%)$ & 0,03 \\
Cicatriz BCG & Negitivo & $11(39,29 \%)$ & $9(16,07 \%)$ & \\
& Positivo & $14(82,35 \%)$ & $39(84,78 \%)$ & 1 \\
Edad (años) & & $31,18 \pm 12,38$ & $31,04 \pm 12,19$ & 0,95 \\
Peso inicial (Kg.) & & $52,96 \pm 7,28$ & $54,65 \pm 7,23$ & 0,51 \\
IMC inicial & & $21,13 \pm 3,43$ & $21,32 \pm 2,60$ & 0,44
\end{tabular}


Tabla $N^{\circ} 2$. Seguimiento de los pacientes del estudio.

\begin{tabular}{lccccc}
\hline \multicolumn{1}{c}{ Variable } & $\mathbf{n}$ & Casos & $\mathbf{n}$ & Controles & $\mathbf{p}$ \\
\hline Peso al primer mes & 14 & $51,61 \pm 7,12$ & 47 & $57,12 \pm 6,99$ & 0,02 \\
Peso al segundo mes & 12 & $53,42 \pm 8,08$ & 49 & $57,38 \pm 7,51$ & 0,18 \\
IMC al primer mes & 11 & $19,68 \pm 1,87$ & 41 & $22,33 \pm 2,49$ & 0,003 \\
IMC al segundo mes & 10 & $19,74 \pm 2,75$ & 42 & $22,38 \pm 2,63$ & 0,01 \\
Frotis positivo al primer mes & 24 & $8(33,33 \%)$ & 54 & $7(12,96 \%)$ & 0,07 \\
Frotis positivo al segundo mes & 23 & $7(30,43 \%)$ & 56 & $2(3,57 \%)$ & 0,04 \\
\hline
\end{tabular}

Tabla $\mathbf{N}^{\circ} 3$. Análisis bivariado y multivariado para fracaso al tratamiento antituberculoso.

\begin{tabular}{|c|c|c|}
\hline \multirow{2}{*}{ Variables } & \multirow{2}{*}{$\begin{array}{c}\text { Análisis bivariado } \\
\text { OR (IC 95\%) }\end{array}$} & \multirow{2}{*}{$\begin{array}{c}\text { Análisis multivariado } \\
\text { OR (IC 95\%) }\end{array}$} \\
\hline & & \\
\hline \multicolumn{3}{|l|}{ Antecedentes: } \\
\hline \multicolumn{3}{|c|}{ Antecedente de tratamiento previo } \\
\hline No & 1 & 1 \\
\hline Sí & $4,89(1,22-19,65)$ & $6,82(1,02-45,70)$ \\
\hline \multicolumn{3}{|c|}{ Seguimiento al primer mes de tratamiento: } \\
\hline \multicolumn{3}{|c|}{ Diferencia de peso entre primer mes y el inicial: } \\
\hline No perdió peso & 1 & \\
\hline Perdió peso & $9,65(2,06-45,10)$ & \\
\hline \multicolumn{3}{|c|}{ Diferencia de IMC entre primer mes y el inicial: } \\
\hline No perdió IMC & 1 & 1 \\
\hline Perdió IMC & $10,65(2,02-56,01)$ & $10,65(2,02-56,01)$ \\
\hline \multicolumn{3}{|l|}{ Resultado de esputo: } \\
\hline Negativo & 1 & \\
\hline Positivo & $3,66(1,10-12,20)$ & \\
\hline \multicolumn{3}{|c|}{ Seguimiento al segundo mes de tratamiento: } \\
\hline \multicolumn{3}{|c|}{ Diferencia de peso entre segundo mes y el inicial: } \\
\hline No perdió peso & 1 & \\
\hline Perdió peso & $10,28(2,31-45,78)$ & \\
\hline \multicolumn{3}{|c|}{ Diferencia de IMC entre segundo mes y el inicial: } \\
\hline No perdió IMC & 1 & 1 \\
\hline Perdió IMC & $10,35(2,00-53,61)$ & $7,08(1,06-47,55)$ \\
\hline \multicolumn{3}{|l|}{ Resultado de esputo: } \\
\hline Negativo & 1 & 1 \\
\hline Positivo & $13,94(2,44-79,60)$ & $25,58(2,10-311,27)$ \\
\hline
\end{tabular}

para asegurar un diagnóstico rápido, es prioritario encontrar pautas para la identificación precoz de pacientes con formas resistentes de tuberculosis (7).

Además de los factores conocidos para el desarrollo de tuberculosis resistente, como abandono de tratamiento, número de tratamientos específicos previos o contacto con un paciente con diagnóstico de TBC-MDR (14,15); se debe considerar factores clínicos y de laboratorio que puedan predecir fracaso a tratamiento. En este estudio, el $84 \%$ de los casos resultaron ser resistentes a isoniazida y rifampicina, requiriendo tratamiento con esquemas estandarizados o individualizados, lo cual aumenta los costos de la Estrategia Nacional. 
Los resultados del presente trabajo muestran que el antecedente de tratamiento específico previo es un factor asociado fuertemente a fracaso a tratamiento que puede alertarnos sobre la posibilidad de fracaso primario o secundario (16). Lamentablemente, en zonas de alta pobreza, la posibilidad de recaída o re-infección es mayor, lo que podría hacer que las tasas de infección por formas resistentes de la enfermedad sean mayores en esas zonas $(11,17)$. Más aún, el hacinamiento secundario a la pobreza podría favorecer la transmisión y dinámica de la tuberculosis MDR.

El seguimiento, valorado a través de la evaluación cotidiana del peso y del índice de masa corporal (IMC) pone de manifiesto la fortaleza de estos marcadores para predecir fracaso a tratamiento específico en forma precoz. Es de importancia notar que, aunque los promedios generales de peso e IMC según los casos y controles aumentan progresivamente, el aumento producido es mayor en los controles. La infección tuberculosa es una enfermedad que está asociada a moderada a severa malnutrición, secundaria a la hiporexia o anorexia producida así como al efecto consuntivo de la enfermedad (18).

Es significativo también, el hecho de que el IMC sea un marcador más fidedigno que el peso para evaluar síndrome consuntivo, ya que muy pocas veces el índice de masa corporal es un valor calculado en la historia clínica de seguimiento del paciente. Sin embargo, parece ser que la variación del IMC es más trascendental para predecir fracaso a tratamiento durante los dos primeros meses de tratamiento, lo cual puede corroborarse por ser la variable que queda en el modelo final del primer y segundo mes de terapia.

El resultado de esputo al segundo mes de tratamiento, pone en claro que este debe ser uno de los factores asociados a fracaso a tratamiento más importantes (19). Sin embargo, el resultado de esputo al primer mes puede ser un factor sugerente de fracaso por su cercanía al nivel de significancia. Aunque el esputo al tercer y cuarto mes de tratamiento muestra una asociación más fuerte, ningún control presentó esputo positivo en esos meses por lo que no se pudo calcular el riesgo asociado (20).

Las limitaciones de este estudio, como el tamaño muestral o el hecho de que los controles solo son considerados curados bajo la definición del programa de control, con los riesgos que esto acarrea $(5,21)$, exige la realización de estudios prospectivos que determinen en forma exacta la validez de estos resultados.
En conclusión, los pacientes que ingresan a tratamiento antituberculoso y que son MDR podrían detectarse bajo estos criterios: antecedente de tratamiento previo, variación del IMC al primer y segundo mes y esputo positivo al segundo mes de tratamiento. En los países en desarrollo, donde las limitaciones económicas traen problemas de infraestructura e imposibilidad de contar con métodos de diagnóstico rápidos de formas MDR de tuberculosis (22), aunado a factores que pueden ocasionar el abandono a tratamiento específico (15); los antecedentes, y la evolución de los patrones clínicos y de laboratorio tienen una importancia capital para la sospecha de pacientes con esta forma de enfermedad, evitando poner en grave riesgo de salud a gran parte de la población.

\section{Correspondencia:}

Antonio Bernabé Ortiz.

Jirón Guandú 3916. Urbanización Las Palmeras.

Los Olivos.

Lima. Perú.

Correo electrónico: 03887@upch.edu.pe

\section{REFERENCIAS BIBLIOGRÁFICAS}

1. Salud Md. Actualización de la doctrina, normas y procedimientos para el control de la tuberculosis en el Perú. Segunda Edición ed. Lima; 2001.

2. ATS. Treatmentof tuberculosis. Morbidity and Mortality Weekly Report. 2003;52(11):1-88.

3. CDC. Treatment of tuberculosis. MMWR 2003;52(11):188.

4. Chavez A, Blank R, Smith M, Bayona J, Becerra M, Mitnick C. Identifying early treatment failure on category I therapy for pulmonary tuberculosis in Lima Ciudad, Peru. Int J Tuberc Lung Dis 2004; 8(1):52-8.

5. Harries A, Gausi F, Chimzizi R, Salaniponi F. Characteristics and outcome of tuberculosis patients whose sputum smears are positive at or after 5 months of treatment. Int J Tuberc Lung Dis 2004;8(3):384-7.

6. Salomon N, Perlman D, Friedmann P, Buchstein S, Kreiswirth B, Mildvan D. Predictors and outcome of multidrug-resistant tuberculosis. Clin Infect Dis 1995;21(5):1245-52.

7. Becerra MC, Freeman J, Bayona J, et al. Using treatment failure under effective directly observed short-course chemotherapy programs to identify patients with multidrug-resistant tuberculosis. Int J Tuberc Lung Dis 2000;4(2):108-14.

8. Yoshiyama T, Yanai H, Rhiengtong $\mathrm{D}$, et al. Development of acquired drug resistance in recurrent tuberculosis patients with various previous treatment outcomes. Int J Tuberc Lung Dis 2004;8(1):31-8.

9. Suarez PG, Watt CJ, Alarcon E, et al. The dynamics of tuberculosis in response to 10 years of intensive control effort in Peru. J Infect Dis 2001;184(4):473-8. 
10. Garcia M, Ponce A, Garcia M, et al. Tuberculosisrelated deaths within a well-functioning DOTS control program. Emerg Infect Dis 2002;8(11):1327-33.

11. Beggs C, Noakes C, Sleigh P, Fletcher L, Siddiqi K. The transmission of tuberculosis in confined spaces: an analytical review of alternative epidemiological models. Int J Tuberc Lung Dis 2003;7(11):1015-26.

12. Al-Moamary M, Black W, Besuille E, Elwood RK, Vedal $\mathrm{S}$. The significance of the persitent presence of acidfast bacilli in sputum smears in pulmonary tuberculosis. Chest 1999;116:726-31.

13. Ramarokoto $H$, Randriamiharisoa $H$, Rakotoarisaonina A, et al. Bacteriological follow-up of tuberculosis treatment: a comparative study of smear microscopy and culture results at the second month of treatment. Int J Tuberc Lung Dis 2002;6(10):909-12.

14. Espinal MA, Kim SJ, Suarez PG, et al. Standard shortcourse chemotherapy for drug-resistant tuberculosis: treatment outcomes in 6 countries. Jama 2000; 283(19):2537-45.

15. Morsy AM, Zaher HH, Hassan MH, Shouman A. Predictors of treatment failure among tuberculosis patients under DOTS strategy in Egypt. East Mediterr Health J 2003 ;9(4):689-701.
16. Small PM, Fujiwara PI. Management of tuberculosis in the United States. N Engl J Med 2001;345(3):189-200.

17. Clark M, Riben P, Nowgesic E. The association of housing density, isolation and tuberculosis in Canadian First Nations communities. Int J Epidemiol 2002;31(5):940-5.

18. Zachariah R, Spielmann MP, Harries AD, Salaniponi FM. Moderate to severe malnutrition in patients with tuberculosis is a risk factor associated with early death. Trans R Soc Trop Med Hyg. 2002;96(3):291-4.

19. Wilkinson D, Bechan S, Connolly C, Standing E, Short GM. Should we take a history of prior treatment, and check sputum status at 2-3 months when treating patients for tuberculosis? Int J Tuberc Lung Dis 1998;2(1):52-5.

20. Zhao FZ, Levy MH, Wen S. Sputum microscopy results at two and three months predict outcome of tuberculosis treatment. Int J Tuberc Lung Dis 1997;1(6):570-2.

21. Finch D, Beaty CD. The utility of a single sputum specimen in the diagnosis of tuberculosis. Comparison between HIV-infected and non-HIV-infected patients. Chest 1997;111:1174-9.

22. Long R. Drug-resistanttuberculosis.CMJ 2000;163 (4): 425-8.

Recibido: 24/11/05

Aceptado para publicación: 07/08/07 